\title{
Recomendaciones en la Atención de Pacientes en Cirugía Maxilofacial Durante la Pandemia de COVID-19 (SARS-CoV-2)
}

\author{
Recommendations in the Care of Patients in Maxillofacial \\ Surgery During the COVID-19 Pandemic (SARS-CoV-2)
}

\author{
Andrés Melián Rivas'; Matthias Wallach Weinstein'; Camila Boin Bakit² \& Rolando Carrasco Soto ${ }^{3,4}$
}

MELIÁN, R. A.; WALLACH, W. M.; BOIN, B. C. \& CARRASCO, S. R. Recomendaciones en la atención de pacientes en cirugía maxilofacial durante la pandemia de COVID-19 (SARS-CoV-2). Int. J. Odontostomat., 14(4):474-480, 2020.

RESUMEN: La nueva enfermedad por coronavirus 2019 (COVID-19) es la última patología de preocupación internacional. Originada en Wuhan, China, se extendió rápidamente a nivel mundial, razón por la cual fue declarada una emergencia de salud pública. Sus síntomas principales son fiebre, tos, dolor de garganta, dificultad respiratoria, fatiga, malestar general y la anosmia, que ha sido incorporada recientemente. Sin embargo, también se han descrito múltiples casos asintomáticos que han alarmado a la población general. Esta enfermedad, se caracteriza por su alta tasa de contagio y su mecanismo de propagación es el contacto cercano entre personas y a través de fluidos corporales como la saliva y secreciones de las vías aéreas. El personal de salud es especialmente vulnerable a la infección debido a su gran exposición a las secreciones oronasales de los pacientes, sobre todo, aquellas especialidades médicas y odontológicas cuyo campo de acción se centra en estas áreas, siendo la cirugía oral y maxilofacial una de ellas, teniendo un alto riesgo de transmisión de SARS-CoV-2. Por lo tanto, es fundamental para este personal, seguir protocolos de prevención y control de infecciones, junto con una correcta anamnesis, examen y diagnóstico de los pacientes que permita establecer una priorización en las atenciones quirúrgicas, disminuyendo la propagación del virus. El objetivo de esta revisión es conocer las recomendaciones básicas para la priorización de pacientes y el cuidado en los procedimientos quirúrgicos por parte del equipo de cirugía maxilofacial durante la pandemia por COVID-19.

PALABRAS CLAVE: COVID-19; Cirugía Maxilofacial, Cirugía Oral, SARS-CoV-2

\section{INTRODUCCIÓN}

En diciembre de 2019, se informó un brote de una enfermedad respiratoria causada por un nuevo coronavirus en Wuhan, China, el cual se expandió rápidamente hacia diferentes partes del mundo (Yang et al., 2020; To et al., 2020). El agente causal fue denominado Coronavirus 2 del Síndrome Respiratorio Agudo Severo (SARS-CoV-2) por el Centro Chino para el Control y la Prevención de Enfermedades. Posteriormente, la enfermedad fue nombrada COVID-19 por la Organización Mundial de la Salud (OMS), siendo declarada una emergencia de salud pública de preocupación internacional el 30 de enero de 2020 (Peng et al., 2020). Hasta el 8 de mayo del 2020 la OMS ha reportado en el mundo 3.862 .676 personas contagiadas y 268.596 fallecidas.
Este virus se transmite de persona a persona a través de gotitas respiratorias, también por contacto cercano, o por contacto directo con sangre y fluidos corporales de pacientes infectados y también indirectamente por contacto con superficies contaminadas, reportándose mayores tasas de contagio que el coronavirus del síndrome respiratorio agudo severo humano (SARSCoV) y el síndrome respiratorio del Medio Oriente (MERS-CoV), siendo, por el contrario, menos letal que estos últimos (Zeng et al., 2020; Wang et al., 2020). Las manifestaciones clínicas incluyen fiebre, tos no productiva, disnea, mialgia, fatiga, además los exámenes complementarios pueden mostrar recuentos leucocitarios normales o disminuidos y también evidencia de neumonía en una radiografía de tórax (Yang et al., 2020).

\footnotetext{
${ }^{1}$ Pasante servicio de Cirugía Oral y Maxilofacial, Hospital San Juan de Dios, Santiago, Chile.

${ }^{2}$ Cirujana Dentista, Facultad de Odontología, Universidad de Chile, Chile.

${ }^{3}$ Cirujano Maxilofacial, Servicio de Cirugía Maxilofacial, Hospital San Juan de Dios, Santiago, Chile.

${ }^{4}$ Cirujano Maxilofacial, Departamento de Cirugía Maxilofacial, Clínica Las Condes, Santiago, Chile.
} 
El personal odontológico, especialmente de los servicios de cirugía oral y maxilofacial es particularmente vulnerable a la infección de COVID-19, tanto por el área de trabajo cercana a las cavidades oronasales y fluidos corporales de los pacientes en la práctica clínica habitual, como por el tipo de instrumentación utilizado (Bali et al., 2020). Sumado a esto, los impredecibles períodos de incubación del virus, los cuales oscilan entre 1 y 14 días pudiendo llegar incluso a 27 días, y su irregular comportamiento, exponen al personal a tratar a pacientes con cuadros infecciosos imperceptibles e incluso pacientes convalecientes (To et al.; Bali et al.). Por lo tanto, los procedimientos de prevención y control de infecciones son particularmente importantes entre los equipos de Cirugía y Traumatología Maxilofacial.

\section{Riesgo de infección en cirugía maxilofacial}

La rápida propagación del virus SARS-CoV-2 planteó un desafío importante para los sistemas de salud en todo el mundo. La tasa de letalidad de este virus ha ido variando en los distintos países y va aumentando a medida que avanza la edad de los pacientes. Si bien se ha descrito una tasa de un $0 \%$ para niños menores de 10 años, aumenta hasta el $1 \%$ en la sexta década de la vida y llega a superar el $20 \%$ hacía la novena década de vida, con una tasa de letalidad promedio entre el 0,3-5,8 \% (Müller et al., 2020; Onder et al., 2020). La primera muerte médica relacionada con COVID-19 fue reportada en Wuhan, China, el 25 de enero de 2020, donde el afectado fue un médico otorrinolaringólogo. En las estadísticas de la epidemia de Wuhan, los trabajadores de la salud representaron el 3,8\% de los infectados, el $14,8 \%$ tenía enfermedad grave y la tasa de mortalidad general fue de 0,6 \%. (Kowalski et al., 2020). Sin embargo, en China se reportó posteriormente que los trabajadores de la salud se ven afectados en el $29 \%$ de los casos, siendo un porcentaje desproporcionadamente alto (Wang et al.; Zimmerman et al.)

La diseminación de persona a persona del virus SARS-CoV-2 se produce principalmente a través de secreciones respiratorias (Wang et al., 2020; Kowalski et al.), en donde existen rutas de transmisión directas (tos, estornudos e inhalación de gotas) así como una transmisión por contacto (membranas mucosas orales, nasales y oculares (To et al.; Meng et al., 2020). Este virus también puede ser transportado a través de aerosoles producidos durante procedimientos terapéuticos (Zimmerman et al.). Se ha reportado una alta carga viral en la cavidad nasal de los pacien- tes infectados, lo que pone en especial riesgo a diversas especialidades médicas a sufrir una infección por SARS-CoV-2, tales como dentistas, otorrinolaringólogos, gastroenterólogos, neumólogos, terapeutas respiratorios, médicos especialistas en enfermedades infecciosas, cirujanos de cabeza y cuello y cirujanos maxilofaciales, reportándose 2 a 3 veces más probabilidades de contraer el virus (Kowalski et al.).

Los cirujanos orales y maxilofaciales deben ser especialmente conscientes de los nuevos desafíos que implica el riesgo de transmisión del virus entre los pacientes y el personal médico, dado que el personal de los departamentos de Cirugía Oral y Maxilofacial es particularmente vulnerable a la infección de COVID19 debido a su exposición cercana a las cavidades orales, nasales y fluidos corporales de los pacientes en la práctica clínica habitual (To et al.; Peng et al.; Yang et al.). Es por esto que durante la pandemia de SARS-CoV-2, la especialidad debe organizar el tratamiento del paciente de tal manera que la transmisión de la infección se reduzca al mínimo y que todas las condiciones de bioseguridad necesarias estén disponibles para proporcionar una adecuada atención al paciente (Zimmerman et al.). Los largos y variables períodos de incubación del virus en pacientes con COVID-19 exponen al riesgo de tratar a pacientes asintomáticos. Peor aún, muchos pacientes que portan el virus pueden ocultar sus síntomas de gripe o pueden estar convalecientes por la enfermedad (Bali et al.). En adición a esto, en China, se realizó un estudio retrospectivo donde se evaluaron 34 pacientes que fueron sometidos a cirugías electivas de distintos niveles de complejidad (desde cirugías de bajo riesgo y baja dificultad técnica, hasta cirugías de alto riesgo con alta dificultad técnica) en la etapa inicial de la pandemia de COVID-19. El estudio demostró que el $44 \%$ de los pacientes terminaron en la UCl posterior a la cirugía (siendo un $26 \%$ habitual en pacientes con COVID-19 sin someterse a cirugía), mientras que $20,6 \%$ de los pacientes fallecieron posterior a la cirugía por complicaciones asociadas a COVID-19 (habitual $<1 \%$ de pacientes con COVID-19 sin someterse a cirugía), siendo la mortalidad de casi 10 a 20 veces mayor. Sin embargo, los autores de este estudio concluyen que los pacientes podrían ser portadores de COVID-19 previo a la cirugía y ésta junto con los diversos mecanismos de respuesta del sistema inmune "activaría" el virus generando los síntomas característicos (Lei et al., 2020). Por estas razones, debe evitarse en lo posible la realización de procedimientos quirúrgicos electivos, en virtud de salvaguardar la salud de los profesionales de la salud y de los pacientes. 


\section{Consideraciones en la atención de pacientes}

El actual brote de COVID-19 ha demostrado que la escasez de recursos de protección personal aumenta el riesgo ya que la atención médica debe enfocarse en pacientes críticos con COVID-19. Lo anterior genera el aplazamiento de cirugías electivas para reducir la presión sobre el sistema de salud (Zimmermann et al.) y las decisiones para realizarlas, deben tener consideraciones médicas y logísticas. En este contexto, es importante evitar una pérdida de recursos al proporcionar el acceso a procedimientos idénticos ofrecidos por diferentes especialidades como, por ejemplo: procedimientos redundantes entre la cirugía maxilofacial y la odontología general. Definitivamente, tiene sentido asignar a la odontología general procedimientos de exodoncia y sus complicaciones relacionadas, como sangrado, tratamiento de abscesos localizados en la cavidad oral, reparación de fístulas oroantrales y tratamientos pulpares. Por otro lado, la cirugía oral y maxilofacial debe enfocarse en el tratamiento de pacientes con infecciones localizadas o difusas de cabeza y cuello, fracturas del macizo craneofacial y trastornos temporomandibulares moderados a severos que solo pueden solucionarse mediante cirugía, postergando procedimientos quirúrgicos electivos como: cirugías reconstructivas de labio leporino, paladar hendido, deformidades craneofaciales y tumores benignos. Esto permitiría utilizar recursos limitados de manera efectiva (Zimmermann et al.; Asociación Peruana de Cirugía Bucal y Maxilofacial, 2020).

En consecuencia, del punto anterior, es fundamental categorizar los procedimientos quirúrgicos en el departamento de Cirugía Oral y Maxilofacial. Lo primero que se debe realizar es una anamnesis relevante a todo tipo de pacientes, que incluya el historial de viajes a lugares afectados por COVID-19, la presencia de síntomas como fiebre, falta de aliento, tos y pérdida del olfato y/o gusto. Las respuestas positivas a cualquiera de las preguntas anteriores deben generar sospecha, y estos pacientes deben ser derivados para realizarles el examen de detección para SARS-CoV2; de confirmarse el contagio deberán recibir tratamiento adecuado según su cuadro clínico (Bali et al.). Desde el punto de vista de la estadía intrahospitalaria de los pacientes, debe existir una organización tal, que cada uno se encuentre lo más aislado posible de otro. Por lo que, en vista de la escasez de camas de hospital disponibles, se debe dar prioridad a las enfermedades que pueden tener efectos adversos significativos en los pacientes sin cirugía, como traumatismos graves y cáncer (Saibene et al., 2020; Zeng et al.).

\section{Categorización de procedimientos en Cirugía Maxilofacial}

Prioridad Baja: Son procedimientos electivos cuya postergación no afecta en el pronóstico de la cirugía. Se incluyen alteraciones y deformaciones del macizo cráneo-maxilofacial, malformaciones craneofaciales que no producen APNEA de sueño o aumento de presión intracraneal, lesiones patológicas benignas, trauma de tejidos blandos sin afectación de estructuras vitales (por ejemplo, laceraciones intraorales), trastornos temporomandibulares leves a moderados, tratamiento de deformidades dento-faciales (cirugía ortognática), reconstrucciones craneofaciales secundarias y dento-alveolares para fines implantoprotésicos, tratamiento de malformaciones de fisuras labio-alveolo-palatinas y biopsias con sospecha de patologías benignas. En estos procedimientos, se recomienda reprogramar los tratamientos quirúrgicos (Zimmermann et al.). Lo importante es evitar las reuniones presenciales, por lo que una alternativa efectiva es comunicarse con los pacientes a través de una consulta telefónica, una videollamada o una consulta en línea (Zimmerman et al.; Yang et al.; Kowalski et al).

Prioridad intermedia: Son aquellos procedimientos quirúrgicos que requieren ser efectuados prontamente, sin embargo, pueden aplazarse teniendo en consideración los posibles riesgos que esto conlleva. Dentro de este grupo encontramos las cirugías de tumores benignos y quistes maxilares, malformaciones craneofaciales que producen APNEA de sueño o aumentos de la presión intracraneal, trastornos temporomandibulares severos y fracturas cerradas sin pérdida de funcionalidad; de hecho, la gran mayoría de las lesiones faciales se pueden evaluar y tratar en una primera instancia por médicos de urgencia (Zimmerman et al.; Edwards et al., 2020; Yang et al.). Se recomienda aplazar estos procedimientos, sin embargo, debe existir un monitoreo constante de los factores de riesgo, los cuales, en caso de ser desfavorables para el paciente, obligarían a realizar los procedimientos quirúrgicos necesarios; para lo cual los pacientes deberán ser sometidos a pruebas de detección de COVID-19. En aquel caso donde el examen resulte negativo, se puede realizar la intervención quirúrgica bajo rigurosas medidas de protección. $\mathrm{Si}$, por el contrario, el paciente resulta positivo, se debe notificar y derivar a su centro de referencia para que realicen su cirugía bajo estrictas medidas de protección, donde se recomienda una evaluación completa del riesgo antes de la admisión, que incluye un recuento sanguíneo completo, bioquímica sérica, radiografía de tórax y evaluación preanestésica. (Yang et al.). 
Prioridad alta: Dentro de este grupo se incluyen aquellas intervenciones urgentes que requieren pronta realización, cuya postergación influiría negativamente en el pronóstico de las afecciones y del paciente. Dentro de este grupo se incluyen biopsias de lesiones potencialmente malignas, cirugías de tumores malignos, trauma de tejidos blandos con afectación de estructuras vitales (tales como el nervio facial, párpados, el sistema lagrimal y la nariz), fracturas cerradas con pérdida de función, abiertas y/o conminutas, infecciones odontogénicas y no odontogénicas profundas de cabeza y cuello que no provocan compromiso de la vía aérea o sepsis de riesgo vital. Los pacientes que requieran este tipo de intervenciones deberán ser sometidos a pruebas de detección de COVID-19 y evaluaciones preoperatorias que incluyen pruebas de laboratorio para SARS-CoV-2 y una tomografía computarizada (TC) de pulmón, con la finalidad de evitar una exposición innecesaria al virus (Zimmerman et al.; Yang et al.). Estos pacientes, como se explicó anteriormente, deberán ser sometidos a un examen de COVID-19, donde un resultado negativo, permitirá realizar la cirugía con las medidas de protecciones adecuadas; mientras que, si el resultado arroja positivo, se deberá realizar la notificación, derivación y manejo por parte del centro de referencia respectivo (Yang et al.; Edwards et al).

Urgencias de riesgo vital: Es indicación de cirugía inmediata aquellas intervenciones de urgencias de riesgo vital, debido a afecciones potencialmente mortales, como hemorragias severas y obstrucciones de las vías respiratorias superiores después de un trauma, tumores o infecciones tanto odontogénicas como no odontogénicas de cabeza y cuello. (Zimmerman et al.; Edwards et al.). Como no es posible realizar un análisis previo de infección por COVID-19 dada la necesidad inmediata de realizar la cirugía, debe tratarse al paciente como un paciente infectado y por consecuencia, el equipo debe cumplir con todos los protocolos estrictos de prevención y control de infecciones además de la práctica de precauciones estándar de rutina para realizar el procedimiento quirúrgico (Yang et al.; Bali et al.). Los procedimientos y algoritmo de atención se resumen en la Tabla I y Figura 1 respectivamente.

\section{Consideraciones en cirugía oral y maxilofacial}

Antes de la cirugía. Antes de llevar a un paciente al quirófano, es ideal realizar una prueba para diagnóstico de SARS-CoV-2, sin embargo, todo paciente de emergencia que no deja tiempo para tomar el examen debe ser tratado como infeccioso, por lo que se deben cumplir los siguientes requerimientos:
- El personal responsable de la transferencia debe usar un respirador N95 o FFP2 con válvula, así como gorra, bata y guantes (Peng et al.; Asociación Peruana de Cirugía Bucal y Maxilofacial).

- El transporte de pacientes hospitalizados debe limitarse estrictamente a los elevadores de transferencia de pacientes hospitalizados para evitar una posible infección cruzada de COVID-19. Los pacientes deben colocarse en habitaciones individuales bien ventiladas, cuyas puertas deben mantenerse cerradas en todo momento y con la entrada y salida limitada al mínimo (Yang et al.).

- Antes de ingresar a la sala de operaciones, cada miembro del personal debe ponerse el equipo de protección personal: además de guantes estériles y protección ocular, deben utilizar batas impermeables, respiradores de alto nivel de protección (N95) con mascarilla quirúrgica encima del respirador, esto último de vital importancia, dado que las máscaras quirúrgicas fueron absolutamente ineficaces para proteger a las personas del humo quirúrgico (Zeng et al.).

- El equipo quirúrgico no debe estar presente en la sala de operaciones durante la intubación, la que debe ser realizada por un anestesista experimentado para limitar el número de intentos y también de una manera que genere la menor tos posible por parte del paciente (Zimmerman et al.). El equipo quirúrgico debe ingresar al quirófano 20 minutos después de la intubación con el equipo de protección adecuado para minimizar la transmisión basada en aerosoles (Kowalski et al.).

- Un enjuague previo al procedimiento podría reducir la carga de coronavirus en la saliva. Se ha demostrado que la povidona yodada tiene una actividad virucida significativa durante aproximadamente tres horas y recientemente se ha recomendado cubrir la cavidad oral y las fosas nasales tanto del paciente como del equipo operativo antes del procedimiento (Bali et al.)

Durante la cirugía.Un quirófano con un entorno de presión negativa es ideal para reducir la diseminación del virus. Una alta frecuencia de cambios de aire reduce la carga viral dentro del quirófano (25 veces por hora) (Kowalski et al). Cada quirófano debe tener su propio sistema de ventilación con un filtro integrado de partículas de alta eficiencia (HEPA). El número de miembros del personal en la sala de operaciones debe limitarse al mínimo (Zeng et al.). 


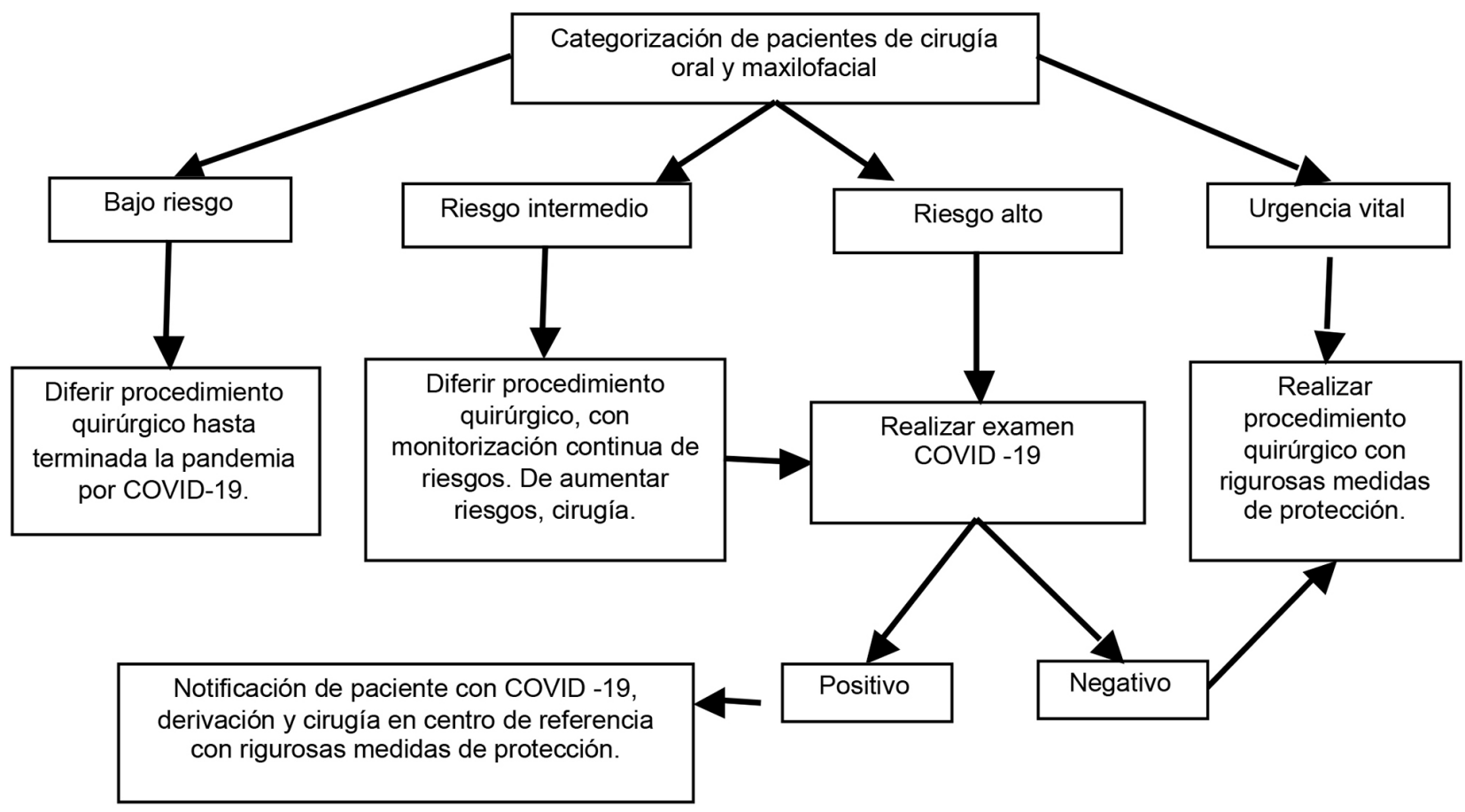

Fig. 1. Algoritmo de atención en cirugía oral y maxilofacial durante la pandemia de COVID -19

Tabla I. Categorización de procedimientos en cirugía oral y maxilofacial durante la pandemia de COVID -19

\begin{tabular}{ll}
\hline Prioridad baja & Cirugía Ortognática \\
& Cirugía de malformaciones craneofaciales que no producen APNEA de sueño o aumento de presión \\
intracraneal & Reconstrucciones craneofaciales secundarias \\
& Trastornos temporomandibulares leves - moderados. \\
& Tratamiento de malformaciones de fisuras labio alveolo palatinas \\
& Trauma de tejidos blandos sin afectación de estructuras vitales. \\
& Biopsias \\
Cirugía de tumores benignos & Cirugía de quistes de los maxilares \\
Prioridad intermedia & Fracturas cerradas sin pérdida de funcionalidad \\
& Cirugía de malformaciones craneofaciales que producen APNEA de sueño o \\
& aumentos de presión intracraneal. \\
& Trastornos temporomandibulares severos \\
& Cirugía de tumores malignos. \\
Prioridad alta & Trauma de tejidos blandos con afectación de estructuras vitales. \\
& Fracturas cerradas con pérdida de función, abiertas y conminutas. \\
& Biopsias de lesiones potencialmente malignas. \\
Infecciones odontogénicas y no odontogénicas profundas de cabeza y cuello sin compromiso de vía & aérea o sepsis. \\
Infecciones odontogénicas y no odontogénicas profundas de cabeza y cuello con compromiso de vía \\
aérea o sepsis \\
Hemorragias severas. \\
Traumas y/o tumores con compromiso de vía aérea. \\
Urgencia riesgo vital
\end{tabular}

Las salas de operaciones de pacientes con COVID-19 deben separarse de las otras para evitar contaminación cruzada, mientras que el flujo de aire contaminado puede minimizarse cerrando todas las puertas al quirófano durante la cirugía, con solo una ruta posible para la entrada y salida. También se recomienda que se use la misma habitación y máquina de anestesia para todos los pacientes con COVID-19 durante la epidemia (Kowalski et al.). 
Durante el procedimiento quirúrgico, se recomienda la presencia de personal con equipo de protección personal estacionado fuera del quirófano en caso de que se necesiten otros medicamentos o insumos (Kowalski et al.). Finalmente, para reducir aún más la posibilidad de infección, los tipos de artículos en la sala de operaciones deben reducirse para tener solo lo indispensable, los artículos desechables deben usarse tanto como sea posible (Zeng et al.; Yang et al.).

Es importante reducir al mínimo la formación de aerosoles considerando que:

- La cauterización eléctrica debe ser evitada o realizarse con la menor potencia posible e idealmente hacerlo con un sistema de evacuación de humo (Zimmerman et al.). Por otra parte, es recomendable dar prioridad al bisturí ultrasónico para reducir el humo quirúrgico en aquellas operaciones que permiten su utilización (Zeng et al.).

- Se debe evitar el enfriamiento excesivo con chorro de agua a piezas de mano, sierras, dispositivos ultrasónicos y dispositivos piezoeléctricos, y aplicar una succión potente para reducir la difusión del humo quirúrgico y los aerosoles (Zeng et al.).

- Si se ha usado instrumental rotatorio, idealmente no volver a utilizar el quirófano hasta transcurridas 3 horas, ya que se ha comprobado que el COVID-19 se mantiene viable en aerosoles durante ese tiempo, aunque reduciendo progresivamente su capacidad infectiva (Asociación Peruana de Cirugía Bucal y Maxilofacial)

Después de la cirugía. Debe transcurrir un intervalo de 15 minutos después de que el paciente haya abandonado el quirófano antes de que puedan comenzar la limpieza y la desinfección. (Zimmerman et al.). Después del procedimiento quirúrgico, la estación de trabajo de anestesia debe esterilizarse. Con respecto a esto, se ha reportado que el uso de un esterilizador de circuito de anestesia (que contenía peróxido de hidrógeno al $12 \%$ ) por dos horas ha sido efectivo. Por otra parte, se ha descrito el uso de desinfectantes para la limpieza el piso del quirófano y las superficies de todo el equipo médico reutilizable como soluciones con contenido de cloro en concentraciones de $500 \mathrm{mg} / \mathrm{L}$ $2000 \mathrm{mg} / \mathrm{L}$ como el hipoclorito de sodio al $1 \%$, también se ha reportado Etanol $70 \%$ o compuestos de amonio cuaternario de quinta generación los cuales han reportado buenos resultados en la inactivación del virus (Kampf et al., 2020; Yang et al.; Kowalski et al.; Asociación Peruana de Cirugía Bucal y Maxilofacial). El instrumental médico reutilizable debe remojarse al menos 30 minutos, idealmente en soluciones con contenido de cloro en concentraciones de $500-2000 \mathrm{mg} / \mathrm{L}$ (como hipoclorito de sodio $1 \%$ ), para luego ser sellados y recogidos en bolsas de desechos médicos desechables de doble capa en la sala de limpieza y así ser enviados al área designada de desinfección. Finalmente, después de limpiar el quirófano, se recomienda apagar el sistema de purificación después de 30 minutos de operación continua de flujo laminar de presión negativa (Kowalski et al.; Asociación Peruana de Cirugía Bucal y Maxilofacial).

\section{CONCLUSIÓN}

Para abordar la pandemia de COVID-19 desde el campo de la cirugía y traumatología maxilofacial de forma adecuada, es fundamental establecer protocolos que permitan priorizar los procedimientos quirúrgicos dependiendo del tipo de patología, de los recursos humanos y de los elementos de protección disponibles para resolver las diferentes afecciones. El objetivo principal es proteger a los pacientes y al equipo odontológico de infecciones innecesarias, evitando la saturación del sistema de salud; siendo imprescindible tomar las medidas de precaución necesarias tanto de infraestructura (unidades ambulatorias, hospitalarias y quirófanos) como de la protección de los pacientes y el personal de salud.

MELIÁN, R. A.; WALLACH, W. M.; BOIN, B. C. \& CARRASCO, S. R. Recommendations in the care of patients in maxillofacial surgery during the COVID-19 pandemic (SARS-CoV-2). Int. J. Odontostomat., 14(4):474-480, 2020.

ABSTRACT: The new coronavirus disease 2019 (COVID-19) is the latest pathology of international concern. Originating in Wuhan, China, it spread rapidly worldwide, which is why it was declared a public health emergency. Its main symptoms are fever, cough, sore throat, shortness of breath, fatigue, general discomfort, and anosmia, which has been recently incorporated. However, multiple asymptomatic cases have also been described that have alarmed the general population. This disease is characterized by its high contagion rate and its propagation mechanism is close contact between people and through bodily fluids such as saliva and airway secretions. Health personnel are especially vulnerable to infection due to their high exposure to patients' oronasal secretions, especially those medical and dental specialties whose field of action focuses on these areas, oral 
and maxillofacial surgery being one of them, having a high risk of transmission of SARS-CoV-2. Therefore, it is essential for these personnel to follow infection prevention and control protocols, together with a correct anamnesis, examination, and diagnosis of patients, which allows prioritizing surgical care, reducing the spread of the virus. The objective of this review is to know the basic recommendations for patient prioritization and care in surgical procedures by the maxillofacial surgery team during the COVID-19 pandemic.

\section{KEY WORDS: COVID-19; Maxillofacial Surgery, Oral Surgery, SARS CoV-2}

\section{REFERENCIAS BIBLIOGRÁFICAS}

Asociación Peruana de Cirugía Bucal y Maxilofacial (ASPECIBUM.) Manual de Atención en Tiempos de COVID 19. Recomendaciones de la Asociación Peruana de Cirugía Bucal y Maxilofacial. Lima, Asociación Peruana de Cirugía Bucal y Maxilofacial, 2020.

Bali, R. K. \& Chaudhry, K. Maxillofacial surgery and COVID-19, The Pandemic !!. J. Maxillofac. Oral Surg., 19(2):159-61, 2020.

Edwards, S. P.; Kasten, S.; Nelson, C.; Elner, V. \& McKean, E. Maxillofacial trauma management during COVID-19: multidisciplinary recommendations. Facial Plast. Surg. Aesthet. Med., 22(3):157-9, 2020.

Kampf, G.; Todt, D.; Pfaender, S. \& Steinmann, E. Persistence of coronaviruses on inanimate surfaces and their inactivation with biocidal agents. J. Hosp. Infect., 104(3):246-51, 2020.

Lei, S.; Jiang, F.; Su, W.; Chen, C.; Chen, J.; Mei, W.; Zhan, L. Y.; Jia, Y.; Zhang, L.; Liu, D.; et al. Clinical characteristics and outcomes of patients undergoing surgeries during the incubation period of COVID-19 Infection. EClinicalMedicine, 100331, 2020. DOI: https://www.doi.org/10.1016/j.eclinm.2020.100331

Meng, L.; Hua, F. \& Bian, Z. Coronavirus disease 2019 (COVID-19): emerging and future challenges for dental and oral medicine. $J$. Dent. Res., 99(5):481-7, 2020.

Müller, O.; Neuhann, F. \& Razum, O. Epidemiologie und Kontrollmaßnahmen bei COVID-19. Dtsch. Med. Wochenschr., 145(10):670-4, 2020.

Onder, G.; Rezza, G. \& Brusaferro, S. Case-fatality rate and characteristics of patients dying in relation to COVID-19 in Italy. JAMA, 2020. DOI: https://www.doi.org/10.1001/jama.2020.4683

Peng, X.; Xu, X.; Li, Y.; Cheng, L.; Zhou, X. \& Ren, B. Transmission routes of $2019-n C o V$ and controls in dental practice. Int. J. Oral Sci., 12:9, 2020.

Saibene, A. M.; Allevi, F.; Biglioli, F. \& Felisati, G. Role and management of a Head and Neck Department during the COVID19 outbreak in Lombardy. Otolaryngol Head Neck Surg., 2020. DOI: https://www.doi.org/10.1177/0194599820917914

To, K.K.-W., Tsang, O. T.-Y., Yip, C. C.-Y., Chan, K.-H., Wu, T.-C., Chan, J. M.-C. \& Yuen, K.-Y. Consistent detection of 2019 novel Coronavirus in saliva. Clin. Infect. Dis., ciaa149, 2020. DOI: https:/ /www.doi.org/10.1093/cid/ciaa149

Wang, D.; Hu, B.; Hu, C.; Zhu, F.; Liu, X.; Zhang, J.; Wang, B.; Xiang, H.; Cheng, Z.; Xiong, Y.; et al. Clinical Characteristics of 138 Hospitalized Patients With 2019 Novel Coronavirus-Infected Pneumonia in Wuhan, China. JAMA, 323(11):1061-9, 2020. DOI: https://www.doi.org/10.1001/jama.2020.1585

Yang, Y.; Soh, H. Y.; Cai, Z. G.; Peng, X.; Zhang, Y. \& Guo, C. B. Experience of diagnosing and managing patients in oral maxillofacial surgery during the prevention and control period of the new coronavirus pneumonia. Chin. J. Dent. Res., 23(1):57$62,2020$.

Zeng, L.; Su, T. \& Huang, L. Strategic plan for management in oral and maxillofacial surgery during COVID-19 epidemic. Oral Oncol., 104715, 2020. DOI: https://www.doi.org/10.1016/ j.oraloncology.2020.104715

Zimmermann, M. \& Nkenke, E. Approaches to the management of patients in oral and maxillofacial surgery during COVID-19 pandemic. J. Craniomaxillofac. Surg., 48(5):521-6, 2020.

Dirección para correspondencia:

Andrés Melián Rivas

Hospital San Juan de Dios

AV. Portales 3239

Santiago

CHILE

Email: andresmelianrivas@yahoo.es

Recibido : 09-05-2020

Aceptado: $12-05-2020$ 